\title{
Erratum to: Euryhalocaulis caribicus gen. nov., sp. nov., a New Member of the Family Hyphomonadaceae Isolated from the Caribbean Sea
}

Wenchao Deng $\cdot$ Yao Zhang $\cdot$ Xiabing Xie $\cdot$

Zihao Zhao Yingnan Fu

Published online: 2 March 2013

(c) Springer Science+Business Media New York 2013

Erratum to: Curr Microbiol

DOI 10.1007/s00284-013-0314-9

In the original version of this paper unfortunately we overlooked a mistake. The corresponding author for this paper is Yao Zhang. Also, there is a typographical error in the title of the original version of this paper (Members). The correct title is "Euryhalocaulis caribicus gen. nov., sp. nov., a New Member of the Family Hyphomonadaceae Isolated from the Caribbean Sea".

The online version of the original article can be found under doi:10.1007/s00284-013-0314-9.

W. Deng $\cdot$ Y. Zhang $(\bowtie) \cdot X$. Xie $\cdot$ Z. Zhao $\cdot$ Y. Fu

State Key Laboratory of Marine Environmental Science, Xiamen

University, Xiamen 361005, People's Republic of China

e-mail: yaozhang@xmu.edu.cn

W. Deng

e-mail: dengwenchao@stu.xmu.edu.cn 